\title{
Fabrication of multi-walled carbon nanotube layers with selected properties via electrophoretic deposition: physicochemical and biological characterization
}

\author{
Aleksandra Benko ${ }^{1}$ Agata Przekora $^{2} \cdot$ Aleksandra Wesełucha-Birczyńska $^{3} \cdot$ \\ Marek Nocuń $^{1} \cdot$ Grażyna Ginalska $^{2} \cdot$ Marta Błażewicz $^{1}$
}

Received: 27 November 2015/Accepted: 9 March 2016/Published online: 23 March 2016

(C) The Author(s) 2016. This article is published with open access at Springerlink.com

\begin{abstract}
The aim of the study was to fabricate and extensively characterize a layer of carbon nanotubes deposited on the surface of titanium, in order to prove that, by selecting proper type of carbon nanotubes and altering different parameters of the electrophoretic deposition process, we are able to obtain products having a different influence on cells-either favouring or inhibiting their survival. In the study, a novel mixture of solvents was used to suspend as-received tubes and then applied in the electrophoretic deposition. High charging capability and high yield of the obtained deposits are promising results when considering up-scaling the process. The surface of the obtained multi-walled carbon nanotubes-coated titanium samples was characterized using SEM, AFM, XPS and Raman microspectroscopy. The carbon nanotube layer showed nanorough topography and was formed of randomly and loosely distributed tubes, and XPS study revealed that there was a significant amount of $\mathrm{C}-\mathrm{O}$ bonds. These properties were found to be favourable to osteoblast survival, spreading and growth.
\end{abstract}

Agata Przekora

agata.przekora@umlub.pl

1 Faculty of Materials Science and Ceramics, AGH University of Science and Technology, A. Mickiewicza 30 Ave., 30-059 Kraków, Poland

2 Chair and Department of Biochemistry and Biotechnology, Medical University of Lublin, Chodzki 1, 20-093 Lublin, Poland

3 Department of Chemical Physics, Faculty of Chemistry, Jagiellonian University, Ingardena 3 St., 30-060 Kraków, Poland

\section{Introduction}

Owing to their unique properties, such as extremely high mechanical properties [1], or good electrical and thermal conductivity [2], multi-walled carbon nanotubes (MWCNTs) are currently proposed to be applied in numerous fields of materials engineering, including biomedicine. Although some applications require usage of a single, separated tube, most of them require forming a micro- or macro-assembly. This can be done either by incorporating the MWCNTs into a polymer matrix or by forming a free-standing film or layer on the substrate of choice. First approach is generally applied when CNTs are aimed to improve the properties of the matrix. However, an introduction of an additional component may hinder the CNTs-CNTs interactions, reducing come of their beneficial properties. Meanwhile, forming purely CNTs-CNTs network may offer the possibility of obtaining a micro- or macro-scale product without reducing the level of inter tube interactions.

Among proposed in the literature methods for fabricating CNT layers (or buckypapers), some of the most popular appear to be printing, drop casting and electrophoretic deposition (EPD). In the first two methods, movement of the particles towards the substrate is induced and governed only by the gravitation forces, while the formation of layer results from the solvent evaporation. Additionally, there is no selectivity where it comes to chemical species being deposited. As a result, highly heterogeneous films are formed, built up not only from the CNTs of assorted sizes, shapes and amount of functional groups, but also from the CNT synthesis by-products. Additionally, orientation of the tubes is random and cannot be controlled and the CNTsCNTs and CNTs-substrate interactions are weak [3]. Contrary to these methods, the EPD is not only facile and 
cost effective, but may also be selective for different type and amount of chemical species, guaranteeing better repeatability of the process and higher quality of the obtained layers. Moreover, this method can yield materials of highly steerable properties - by altering the type of a solvent, voltage, distance between the electrodes or time of deposition, one can obtain materials that are either dense or holey, strongly or weakly adhered to the surface of the substrate [3, 4]. Thus, requirements for different applications, including biomedical, can be met.

Proper solvent selection is an important step in planning the EPD experiments. Usually, a mixture of organic solvents or water is applied. Water assures high yields of deposits but at the same time, its hydrolysis compromises the quality of deposits. On the other hand, organics yield more uniform deposits, but much higher voltages are required and low deposition rate is observed [4]. Thus, a mixture of organics (based on Du's work, acetone and ethanol were chosen [5]) with a small addition of water should be expected to provide the most satisfactory results. To provide higher repeatability of the process, commercially available, $\mathrm{OH}$-functionalized CNTs were used with no additional chemical treatment. This is also a novelty in the field of the EPD of the CNTs-majority of scientists suggest initial oxidation that introduces functional groups to the CNT walls. In this approach, it is very hard to obtain reproducible results, and we believe that this is an origin of numerous scientific discrepancies concerning some of the basic CNT properties, including biocompatibility.

In our recent study, we reported fabrication of welladhered to each other, dense CNT layers that are highly conductive, non-toxic and in the same time inhibitive towards osteoblast proliferation. We hypothesized that large share of carboxyl groups retarded osteoblast spreading and growth [6]. On the other hand, in our different study, we found that fibroblasts are virtually insensitive for CNT surface chemistry and topography, suggesting that there is some other quality of CNT layers that has a major influence on fibroblasts and overcompensates for other properties. This quality, however, is yet to be defined [7]. In the following study, we present results complementary to our previous works. We reveal that it is also possible to fabricate CNT layers that are supportive for osteoblast adhesion and hypothesize that this type of material, due to its biomimetic properties, may exhibit an enhanced bioactivity, as already reported in some of the studies [8], and thus may be considered as a permanent implant in biomedical applications.

In the first step of the study, a stable CNT suspension was prepared and used in the EPD. While depositing the layers, we monitored the current density flow to obtain information about the suspension stability and deposition kinetics. Morphology of the fabricated layer was evaluated using SEM, where both the surface and the cross-section were observed, giving some information about the thickness of the layer. Topography of the layers was evaluated by an atomic force microscope (AFM). Afterwards, Raman spectroscopy and X-ray photoelectron spectroscopy were used to evaluate crystallinity, purity, type and amount of functional groups of both the deposited CNTs and the starting material. This shed some light on the potential selectivity of the EPD process. Consecutively, indirect and direct contact cytotoxicity tests were performed as well as osteoblast morphology on tested surface was analysed in order to assess the biocompatibility of the fabricated CNT layer. In vitro experiments were conducted using human osteoblasts as due to their biomimetic properties, CNTs appear to be promising materials for bone regeneration. Finally, the biocompatibility results were correlated with physicochemical properties of the sample and influence of some of the basic material properties on its biocompatibility was established.

\section{Materials and methods}

\subsection{Carbon nanotubes and titanium}

Multi-walled carbon nanotubes, functionalized with $\mathrm{OH}$ groups (CNTs-OH), were purchased from the Nanostructured \& Amorphous Materials, Inc., USA. Vendor-provided characteristics can be found on the producer site [9].

Sixty squares with side lengths of approx. $10 \mathrm{~mm}$ were cut from titanium plate. The samples were placed for 10 min in ultrasonic bath with acetone and ethanol, consecutively, in order to remove any organic residues. Afterwards, the samples were treated for $30 \mathrm{~s}$ with $5 \%$ fluoric acid, resulting in HF-treated titanium platelets (Ti). The reaction was stopped by the thorough washing with distilled water, until the $\mathrm{pH}$ of the washed over liquid was neutral. Apart from dissolving the titanium dioxide, $\mathrm{HF}$ is also expected to increase the surface area of the material, increase adhesion of the layer and improve biocompatibility of final product $[10,11]$. All reagents were purchased from Avantor Performance Materials Poland, Gliwice.

Approx. $70 \mathrm{mg}$ of the CNTs-OH were dispersed in the mixture of $15 \mathrm{~mL}$ of $96 \%$ vol. ethanol, $5 \mathrm{~mL}$ of acetone and $4 \mathrm{~mL}$ of distilled water, with an aid of an ultrasonic processor Vibra-cell, type VCX 130 from Sonics \& Materials, Inc., equipped with a 6-mm probe. The resulting suspension is referred to as the CNTs-OH_ea throughout the article.

\subsection{Electrophoretic deposition}

The CNTs-OH_ea suspension was used during the EPD process. The set-up consisted of a DC power source 


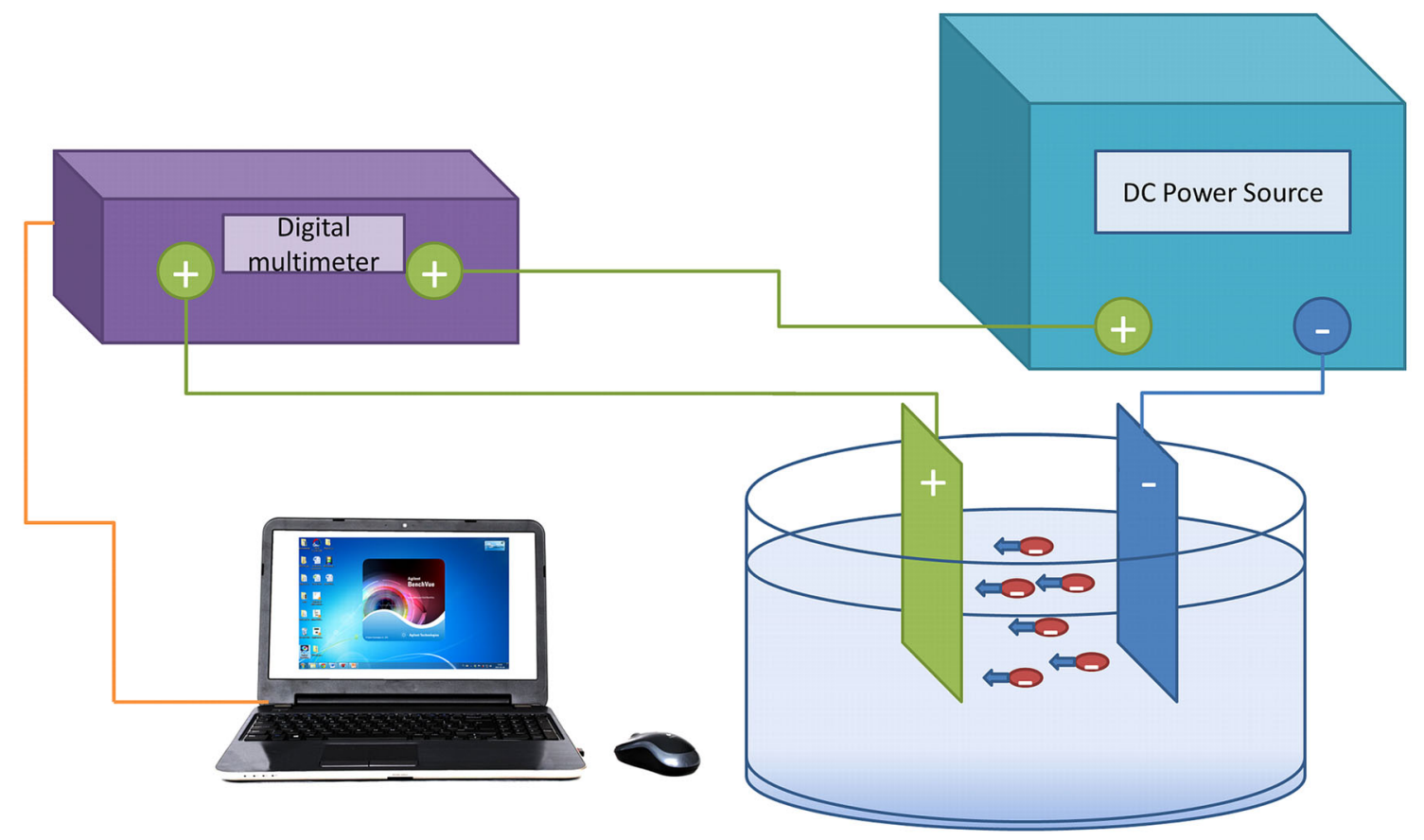

Fig. 1 Electrophoretic deposition set-up

(EL561R from ThurlbyThandar Instruments Limited, UK), a multimeter (34405A, Agilent, USA) connected to a computer, and one stainless steel counter electrode, placed in an electrolytic bath-scheme is presented in Fig. 1. The process was carried out with applied voltage of $30 \mathrm{~V}$, for $10 \mathrm{~s}$. Distance between the titanium platelet and counter electrodes was $5 \mathrm{~mm}$. During the deposition, current magnitude was recorded using BenchVue 1.1 software. After the successful deposition, samples were left to dry in room atmosphere conditions. Accordingly fabricated materials were subsequently washed with ethanol in order to remove CNT species that were not bonded to the surface of titanium. Before the experiments, tested samples were sterilized in $70 \%$ ethanol solution followed by the exposure to UV light up to $1 \mathrm{~h}$ under the hood.

\subsection{Physicochemical characterization}

\subsubsection{Scanning electron microscopy}

All SEM measurements were performed using ultra-highresolution scanning electron microscope-Nova NanoSEM 200, manufactured by FEI Europe Company. The measurements were performed in low vacuum conditions, using a Helix detector and an accelerating voltage of 10, 15 or $18 \mathrm{kV}$. Dimensions of the tubes and the thickness of the layer were evaluated using Visio 2010 software. A total of 20 (CNT diameters) or 10 (thickness of the layer) independent measurements were performed, and the results were expressed as means \pm standard deviations (SD).

\subsubsection{Atomic force microscopy}

MultiMode 8 Bruker microscope, operating in a tapping mode, equipped with antimony-doped silicon tips (spring constant $=40 \mathrm{~N} / \mathrm{m}$ ) was used to evaluate the topography of both titanium and the deposited layer.

\subsubsection{Raman microspectroscopy}

Raman spectra were collected using Renishaw inVia spectrometer, and the Raman light was dispersed by a diffraction grating with 2400 grooves $/ \mathrm{mm}$. The samples were excited with an argon ion laser using excitation wavelength at $514.5 \mathrm{~nm}$. The laser power $(\sim 1-3 \mathrm{~mW}$ at the sample surface) was kept well below the level that causes damage to the sample.

\subsubsection{X-ray photoelectron spectroscopy}

Evaluation of the elements present on the surface of the material, together with their chemical states, was performed using X-ray photoelectron spectroscopy (XPS) (Vacuum Systems Workshop Ltd., England). As the excitation source, $\mathrm{Mg}$ Ka X-ray radiation with $200 \mathrm{~W}$ energy was used. Electron energy analyser was set to FAT mode 
with pass energy $22 \mathrm{eV}$. Depth of analysis was about $5 \mathrm{~nm}$. The average spatial resolution of the applied system is $3 \mathrm{~mm}$, and thus a single measurement is assumed to be representative for the whole sample. Calibration of the spectra was performed by assuming binding energy of $\mathrm{C} 1 s$ to be always $284.6 \mathrm{eV}$. Spectral analysis was performed using XPS Peak 4.1 software. Quantitative evaluation of components with different binding energies was performed based on peaks' areas.

Both XPS and Raman Spectroscopy tests were performed on Ti-CNTs-OH sample and the starting material, $\mathrm{CNTs}-\mathrm{OH}$, in order to evaluate whether the EPD process favours deposition of the CNTs of different structural properties and to provide information about the chemical composition of the CNTs being deposited.

\subsection{In vitro cell culture experiments}

\subsubsection{Live/dead staining}

In vitro experiments were carried out using hFOB 1.19 cell line (normal human foetal osteoblasts), obtained from ATCC (American Type Culture Collection). The cells were cultured according to ATCC recommendation as it was described earlier [6]. To assess the cytotoxicity of tested samples ( $\mathrm{Ti}$ and $\mathrm{Ti}-\mathrm{CNTs}-\mathrm{OH})$ in direct contact with the cells, human osteoblasts were seeded directly on the specimens as described previously [6] and after 24and 48-h incubation stained using Live/Dead Double Staining Kit containing calcein-AM and propidium iodide (PI) dyes (Sigma-Aldrich Chemicals) in accordance with the manufacturer protocol. Stained live (green) and dead (red) cells were observed under fluorescence laser scanning microscope, using two-dimensional scan technique (Olympus Fluoview IV81 equipped with FV1000 laser scanner).

\subsubsection{Colorimetric cytotoxicity evaluation}

Indirect cytotoxicity evaluation was performed using fluid extracts of the samples and colorimetric WST-8 test (Sigma-Aldrich Chemicals) as it was described earlier [6]. Briefly, osteoblasts were seeded on polystyrene 96-multiwell plates and then exposed to the extracts of Ti-CNTs$\mathrm{OH}$ and unmodified $\mathrm{Ti}$ samples, which were prepared according to ISO 10993-5 [12]. Polystyrene extract (PS) served as negative control of cytotoxicity, whereas $0.1 \%$ phenol solution served as positive control. After 24- and 48-h incubation with the extracts, WST-8 test was performed in accordance with the manufacturer protocol to evaluate cell metabolic activity via measurement of mitochondrial dehydrogenases activity. Full details on the experimental cell culture procedures can be found in our previous study [6]. The results obtained with WST- 8 test were expressed as mean values \pm standard deviation (SD). The unpaired $t$ test was performed to assess statistical differences $(P<0.05)$ between tested samples and control group (GraphPad Prism 5, version 5.03 software).

\subsubsection{Cell morphology evaluation}

To assess osteoblast morphology on the tested samples ( $\mathrm{Ti}$ and Ti-CNTs-OH), hFOB 1.19 cells were seeded directly on the specimens and after 48-h incubation stained using AlexaFluor635phalloidin (Invitrogen) for F-actin filaments labelling and Hoechst 33342 dye (Sigma-Aldrich Chemicals) for nuclei counterstaining. The staining procedure was described previously [6]. Stained cells were observed under fluorescence laser scanning microscope.

\section{Results and discussion}

\subsection{Current density flow during the EPD}

In order to prove the applicability of the chosen solvent composition, current density was recorded during the deposition. Representative curves for three separate depositions are presented in Fig. 2. The curves were very similar to each other, proving high repeatability of the process and stability of the CNT suspension. During the deposition, the current density flow was found to increase rapidly when the power source was turned on, proving charging capability of the applied dilution. After an initial rapid increase, the current density value was stabilized at approx. $0.5 \mathrm{~mA}$. Relatively small value of the current density minimizes the risk of suspension heating and electrochemical attack of the deposit, while its high stability during the deposition is expected to provide consistent kinetics of the deposition [4] yielding an uniform assembly [13].

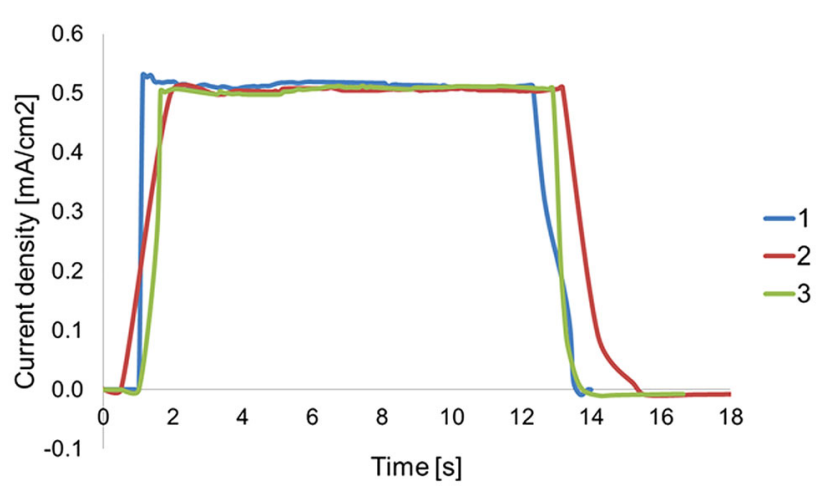

Fig. 2 Current density versus time graphs recorded for three distinctive samples during the EPD process 


\subsection{Physicochemical evaluation}

\subsubsection{Scanning electron microscopy}

Morphological evaluation of the layers was performed via SEM imaging with no thermal spraying of conductive layer. Low magnification observations were performed on both the titanium and the deposited layers, and the results are given in Fig. 3. It can be seen that the CNTs evenly covered the surface of titanium, mimicking its morphology - after the deposition, the grain boundaries of titanium were still clearly visible, as shown in Fig. 3d. The surface of the Ti-CNTs-OH was found to be composed of a layer of uniformly distributed CNTs, covered with randomly but evenly distributed larger agglomerates (as seen in Fig. 3b). Presence of agglomerates suggests deposition from the flocculated state, as already suggested in our previous study [7]. Observation of the layer under higher magnification revealed that the CNTs thoroughly covered the titanium surface and the layer was formed of randomly oriented MWCNTs (Fig. 4a). The layer appeared to be poorly organized, and CNTs gave rather scattered structure (Fig. 4b). At the same time, no large pores in the structure were observed, indicating that the amount of water was small enough to prevent the gas evolution caused by water hydrolysis at the electrodes [4] and the ethanol/acetone ratio was well chosen [5]. Diameter of the CNTs present on the surface of the Ti-CNTs-OH ranges between 10 and $35 \mathrm{~nm}$, with a mean of $23.2 \pm 6.5 \mathrm{~nm}$. After observing the surface of the layer, the sample was cut using steel plate scissors and placed perpendicularly under the microscope. In Fig. 4c, d, the in-depth morphology of the layer was observed. The layer was found to be relatively thick (mean thickness of $4.64 \pm 0.9 \mu \mathrm{m}$ ), suggesting that the deposition rate was high, possibly due to high dielectric constant of the solvents mixture and high mobility of the particles in the applied solution. The CNTs in the layer appeared to be uniformly distributed throughout the layer (Fig. 4c), exclusive of the inner most surface, where the CNT packing was observed in such an extension that it was hard to differentiate between neighbouring tubes (Fig. 4d). Indepth heterogeneity of the CNT distribution may be induced by the building up pressure of the subsequently depositing CNTs, increasing density and favouring optimal packaging at the interface between the tubes and the metal. Meanwhile, uniform distribution further from titanium substrate suggests that the suspension was stable during the deposition, as observed via current density measurements.
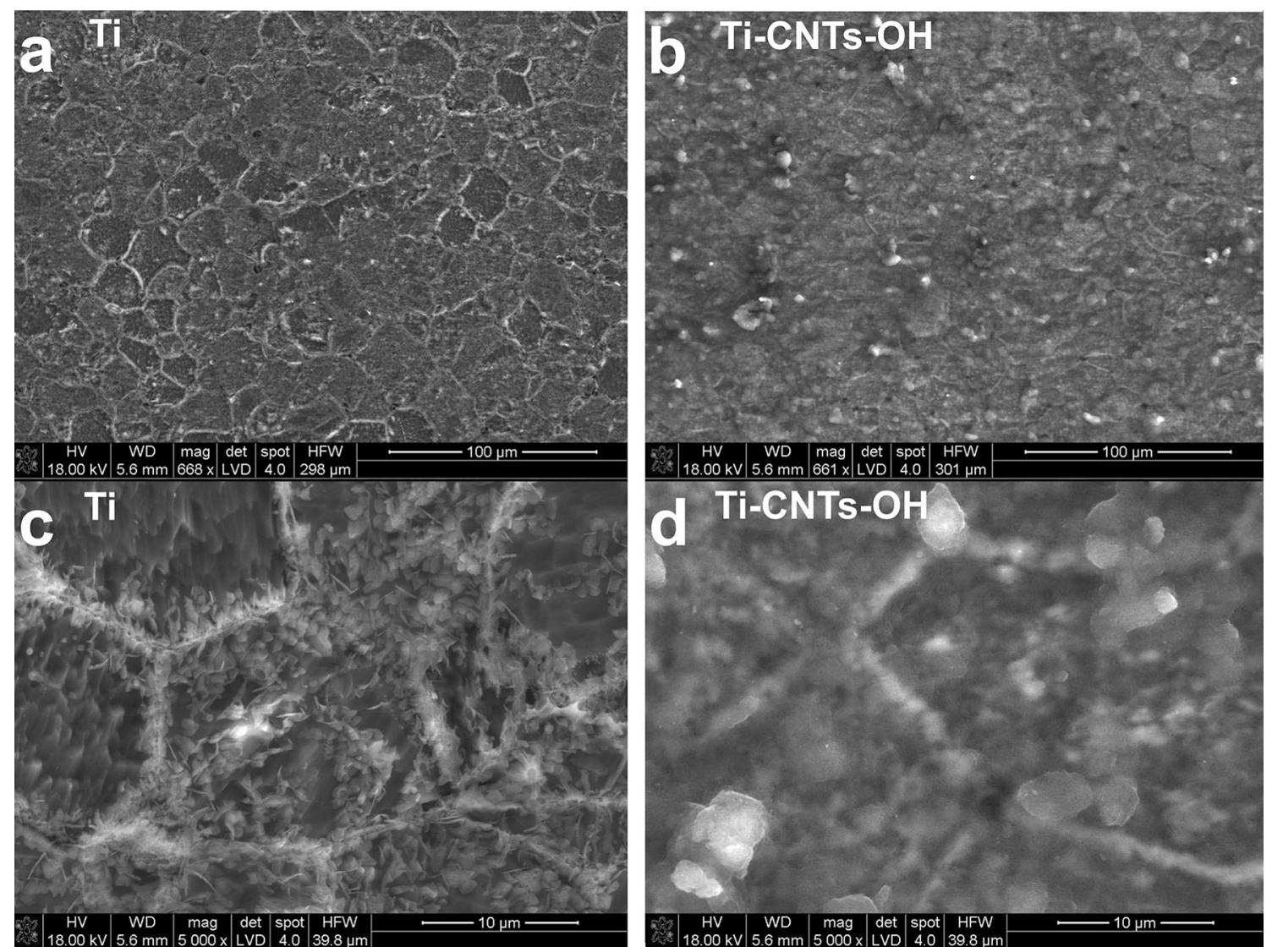

Fig. 3 SEM images obtained at low magnifications for comparison of surface morphologies of Ti (a, c) and Ti-CNTs-OH (b, d) samples 

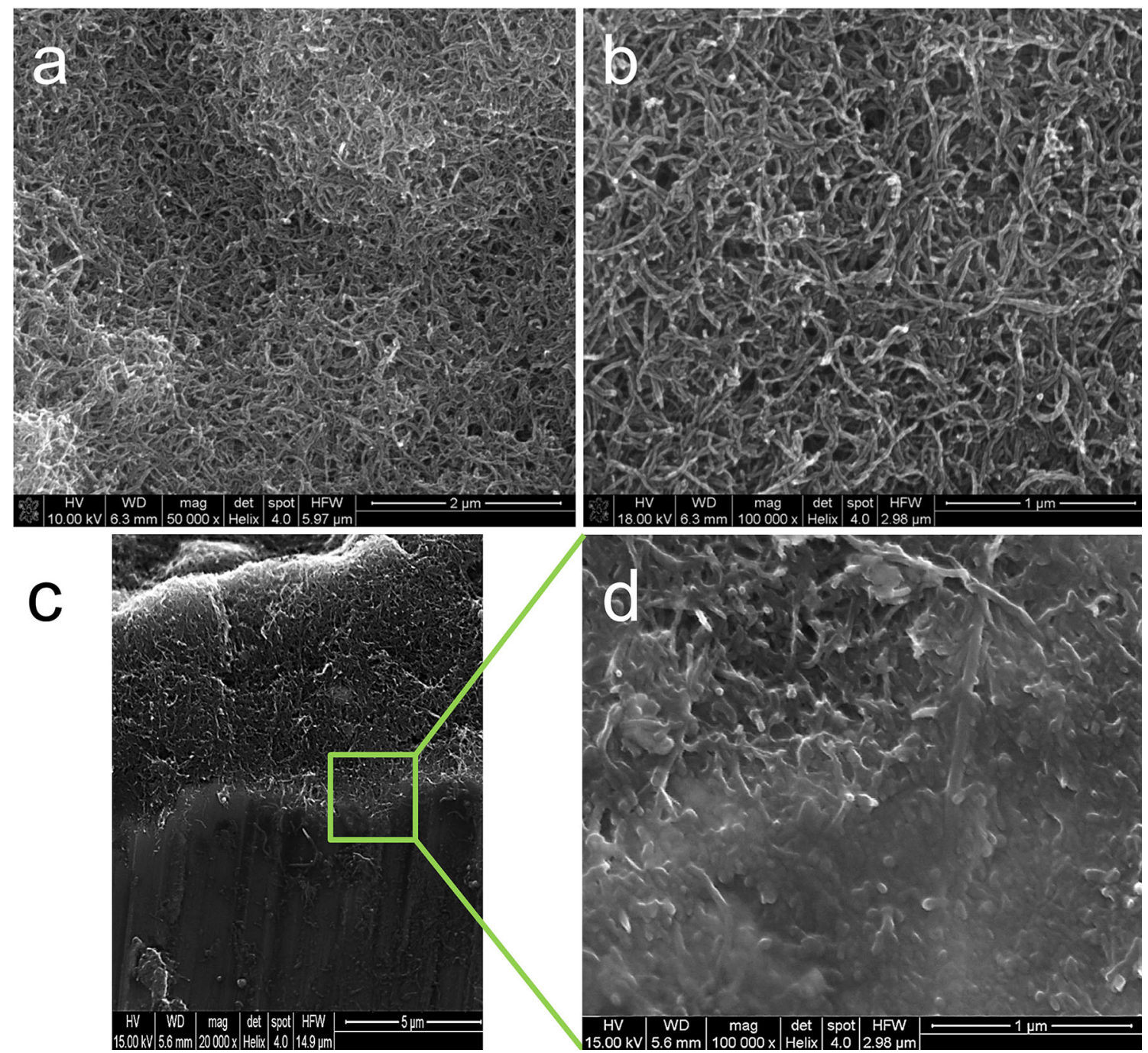

Fig. 4 SEM evaluation of the Ti-CNTs-OH sample; surface images obtained at magn. $50,000 \times(\mathbf{a})$ and magn. 100,000× (b); cross-section images obtained at magn. $20,000 \times(\mathbf{c})$ and magn. $100,000 \times$, focus at the inner most layer $(\mathbf{d})$

\subsubsection{Atomic force microscopy}

Results obtained with the AFM are presented in Fig. 5, with increasing magnification. It can be seen that the surface of titanium had a micro-scaled roughness (Fig. 5a), attributed to the presence of grains and most likely increased during a HF treatment. Deposition of CNTs was found to smooth the surface, reducing its anisotropy and making it more uniform in the micro-scale (Fig. 5b). At the same time, presence of CNTs introduced an additional nanotopography (nanoroughness) to the surface of the material (Fig. 5d). As suggested in the literature, nanotopography might influence the process of focal adhesion, improving or worsening cell adhesion and proliferation by increasing surface area and forcing different integrin orientation and/or agglomeration [14]. In Fig. 5a, b, presence of agglomerates was revealed, which is in good agreement with the results obtained with SEM (Fig. 3) and suggests deposition from the flocculated state.

\subsubsection{Raman microspectroscopy}

The first-order region $\left(1100-1800 \mathrm{~cm}^{-1}\right)$ of all the obtained spectra is given in Fig. 6. For all of the carbonaceous species, this region contains the most informative and prominent bands: the $\mathrm{G}$ band $\left(\sim 1580 \mathrm{~cm}^{-1}\right)$, attributed to carbon atoms within the polyaromatic structure, and the $\mathrm{D}$ band $\left(\sim 1350 \mathrm{~cm}^{-1}\right)$, which arises from the presence of defects. $G$ band is specific for all graphiticbased materials and is ascribed to the crystallinity and pristine arrangement of atoms [15], whereas the D band, also called the defect band, arises from the breathing mode of the carbon rings, which become Raman active at the borders of the crystallite areas due to the loss of their 
Fig. 5 Surface topography evaluation of $\mathrm{Ti}(\mathbf{a}, \mathbf{c})$ and $\mathrm{Ti}-$ CNTs-OH $(\mathbf{b}, \mathbf{d})$ performed via AFM; images show microtopography $(\mathbf{a}, \mathbf{b})$ and nanotopography $(\mathbf{c}, \mathbf{d})$ of the tested samples $\mathbf{a}$
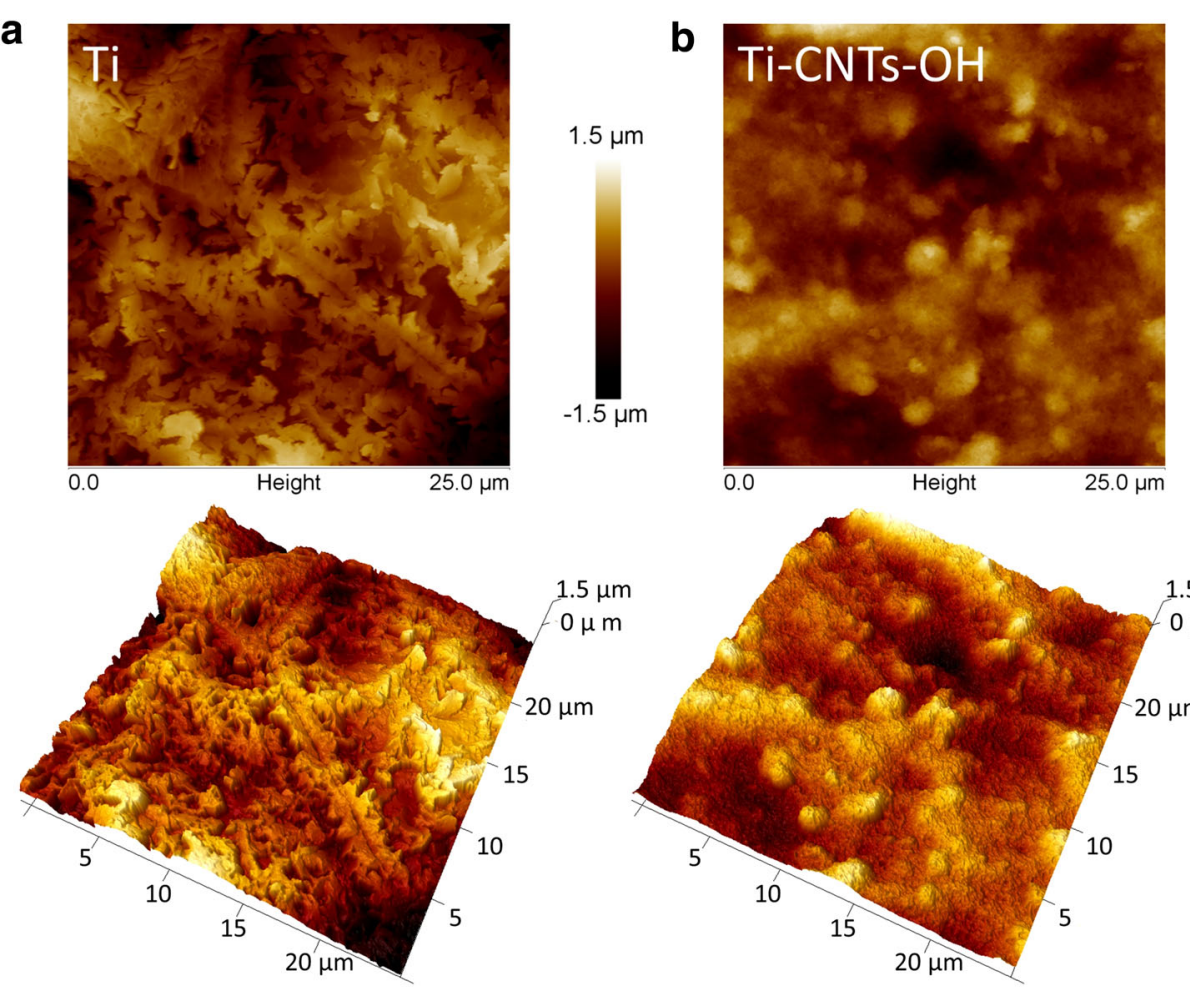

C
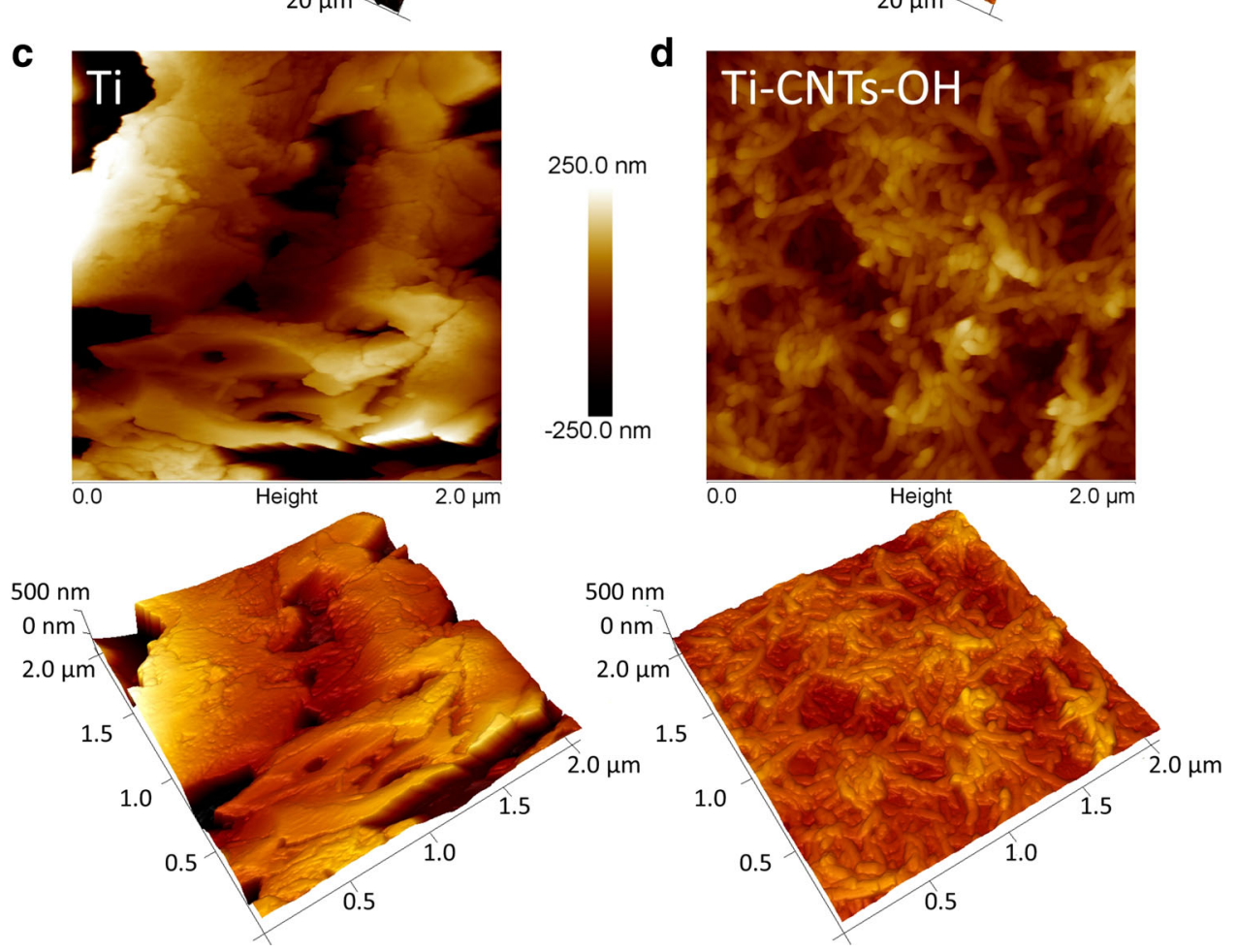

translational symmetry $[16,17]$. This band originates from the double resonances, involving phonons and defects $[16$, 18], and is commonly used to evaluate the level of disorder in carbon materials (CM), e.g. vacancies or defects on CNT walls. Spectrum of poorly organized CM additionally reveals other bands, one of which is the weak shoulder of the $\mathrm{G}$ band, the $\mathrm{D}^{\prime}$ band, arising from the double resonance feature of the in-plane defects and heteroatoms [19-22].

Figure 6 shows Raman spectrum of the as-received CNTs-OH placed on the silicon wafer (A) and spectrum of 


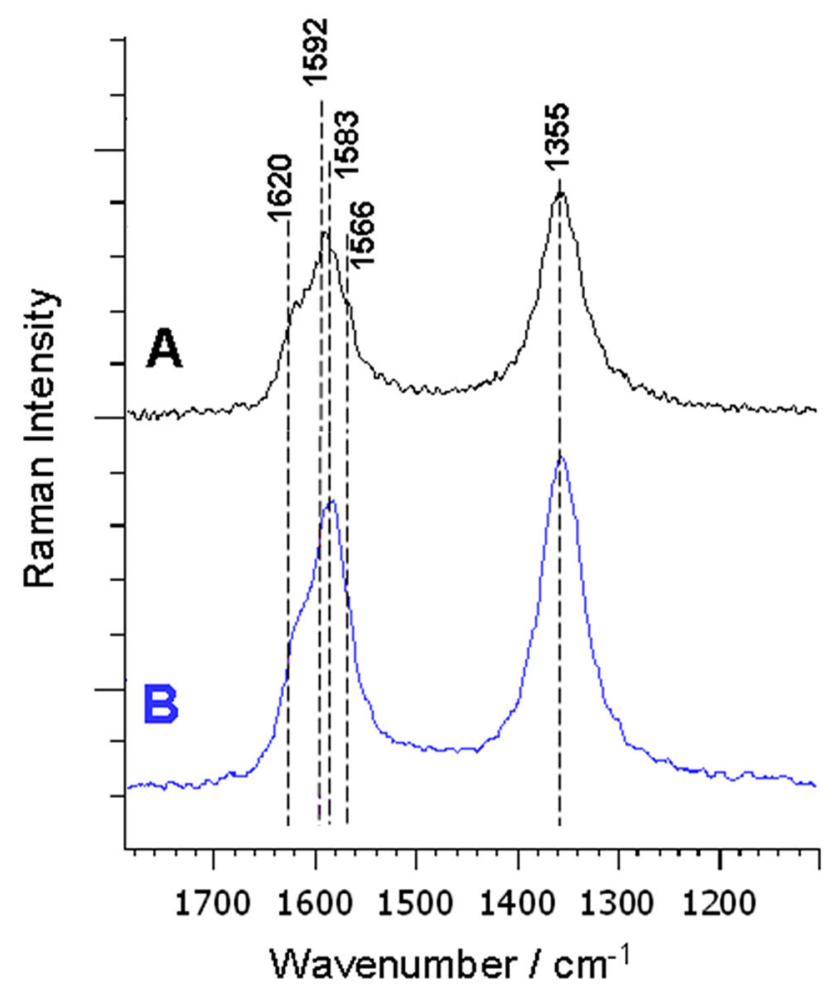

Fig. 6 Raman spectra using excitation line of $514.5 \mathrm{~nm}$, region $1800-1100 \mathrm{~cm}^{-1}$; $a$ CNTs-OH, placed on a silicon wafer; $b$ Ti$\mathrm{CNTs}-\mathrm{OH}$; the spectra were offset for better clarity

Ti-CNTs-OH sample (B). In all of the given spectra, two distinctive bands can be found: the $\mathrm{D}$ band and the $\mathrm{G}$ band. Additionally, a high-frequency $\mathrm{D}^{\prime}$ shoulder of the $\mathrm{G}$ band $\left(\sim 1618 \mathrm{~cm}^{-1}\right)$ was visible in all the spectra, indicating presence of the defective graphite-like structures.

Bands assignments and quantitative evaluation of CNT crystallinity were collected in Table 1. Quantitative assessment was done by calculating the $R 1 \quad\left(I_{\mathrm{D}} / I_{\mathrm{G}}\right)$ parameter, which is a relative integral intensity ratio and provides information about the proportion between the defected and crystalline structure within the tested sample. The higher the $R 1$ ratio is, the lower is the level of crystallinity of the tested sample [15, 20, 23-25]. Basing on the $R 1$ ratio, no significant differences in the structure of the CNTs were observed and the CNTs in the layer were found to have similar level of crystallinity to the starting material. However, when considering the band absorption wavelength, some changes can be found. For the Ti-CNTs-OH, a significant downshift of both $D$ and $G$ bands was observed, probably as a manifest of the residual stress present in the layer and as a consequence of relatively strong CNTs-CNTs interactions [26]. While being deposited, the CNTs were strained by interacting both with the substrate and with each other, elongating the chemical bonds, weakening their strength and causing less loss in energy of the Raman scattered light. As a consequence, a downshift of the bands can be observed [27]. Although some of the very recent studies gave some input in correlating the strain with the high-frequency Raman modes downshift in the CNTs, quantification of stress in MWCNTs is still impossible at this time [28].

\subsubsection{X-ray photoelectron spectroscopy}

All of the obtained spectra consist of two significant photoelectric lines: one is attributed to the carbon $(\mathrm{C} 1 s$ with binding energy of $284.6 \mathrm{eV}$ ) and the other to the oxygen (O1s with binding energy of $532.9 \mathrm{eV}$ ) atoms in the sample. In both samples, different shapes of spectral lines of both atoms were observed (Fig. 7a, b, respectively) and thus presence of different amount of various functionalities was expected. In order to prove that, deconvolution of the bands was performed. Obtained quantitative results are presented in Fig. 7c, d. During the deposition, the amount of the oxygen atoms was observed to change: from the starting material with an average amount of $9.5 \%$, less oxidized CNTs $(7.6 \%)$ were found in the outer surface of the layer. The reason for that might be interactions between some of the functional groups during the deposition, inducing removal of the oxygen.

In both spectra, deconvolution of the $\mathrm{C} 1 s$ band (Fig. 7c) revealed presence of two main peaks: one with B.E. of $284.6 \mathrm{eV}$, which is attributed to carbon atoms in the $\mathrm{sp}^{2}$ hybridization [29-32], forming subsequent graphitic layers of the MWCNTs; and the other, at $285.8 \mathrm{eV}$, arising from the presence of various defects in the structure of the tubes, which may also be the places where the hydroxyl and ether functional groups were attached [32, 33]. It can be observed that during the deposition, the amount of defects (and possibly also $\mathrm{OH}$ and $\mathrm{C}-\mathrm{O}-\mathrm{C}$, groups) increasedfrom $17.5 \%$ for the CNTs-OH to $30 \%$ in the case of $\mathrm{Ti}-$ $\mathrm{CNTs}-\mathrm{OH}$. This might be explained either by a selectivity of the process, favouring deposition of the CNTs with more defects (or functional groups), or by the interactions of the neighbouring tubes, leading to a formation of new defects.

Third observable peak was found to have a B.E. of approx. $287 \mathrm{eV}$ for the CNTs- $\mathrm{OH}$ and $287.5 \mathrm{eV}$ for the Ti-
Table 1 Assignments of Raman bands $\left[\mathrm{cm}^{-1}\right]$ observed for CNT coating films

\begin{tabular}{lllll}
\hline Types of CNTs film coatings & $\mathrm{D}^{\prime}\left[\mathrm{cm}^{-1}\right]$ & $\mathrm{G}\left[\mathrm{cm}^{-1}\right]$ & $\mathrm{D}\left[\mathrm{cm}^{-1}\right]$ & $R 1$ \\
\hline CNTs-OH & 1624 & 1589 & 1354 & 1.28 \\
Ti-CNTs-OH & 1617 & 1583 & 1354 & 1.25 \\
\hline
\end{tabular}




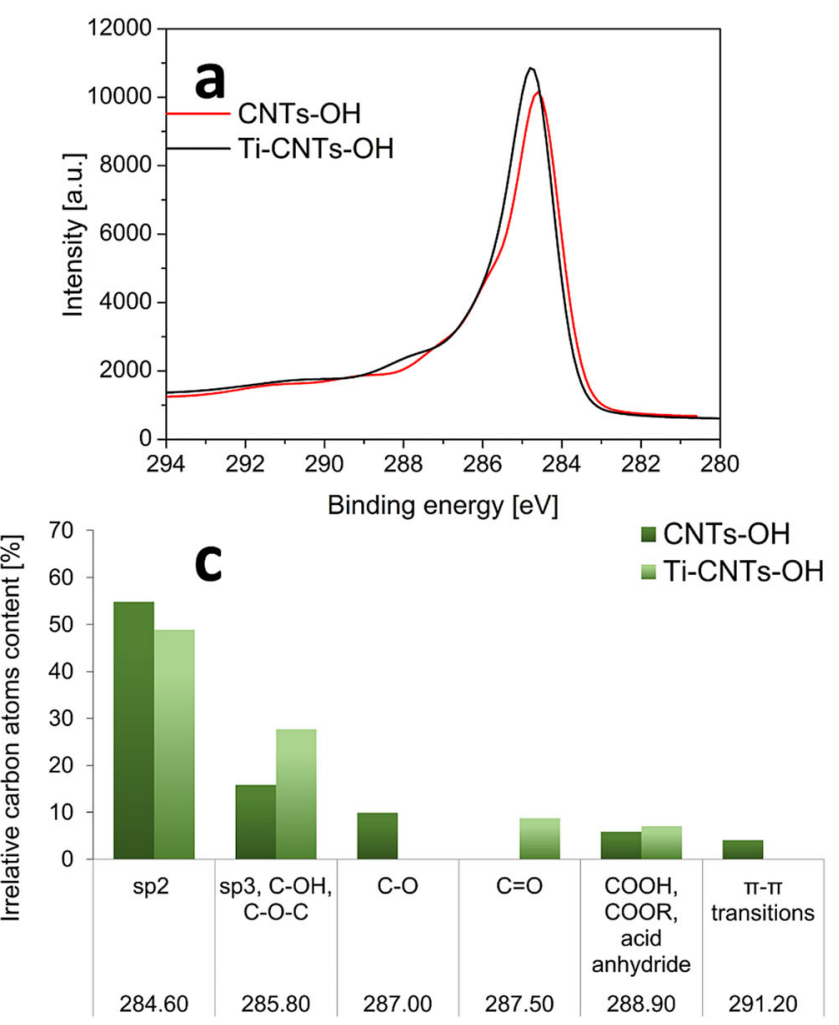

Fig. 7 High-resolution XPS spectra of the $\mathrm{C} 1 s(\mathbf{a})$ and $\mathrm{O} 1 s(\mathbf{b})$ bands peaks found in the spectrum of the CNTs- $\mathrm{OH}$ and Ti-CNTs-OH; the bands are maximized for better clarity; quantitative and qualitative

$\mathrm{CNTs}-\mathrm{OH}$. Higher binding energies suggest presence of double bonds [32] between carbon and oxygen atoms instead of a single bond $[34,35]$. In both samples, another component of the carbon band was connected to the presence of the carboxyl functional groups [32, 36], with B.E. of 288.9 and $290.2 \mathrm{eV}$ for the CNTs-OH and Ti-CNTs$\mathrm{OH}$, respectively. Higher value of the B.E. might suggest that part of the functional groups underwent a recombination, resulting in formation of the carbonates and/or acid anhydride [32]. In the Ti-CNTs-OH, the relative amount of $\mathrm{COO}$ groups was higher (7.7 \%, compared to $6.5 \%$ ), implicating that during the deposition, CNTs with higher amount of carboxyl species were favoured over the less carboxylated ones, possibly thanks to the higher electric charge.

In the case of the CNTs, a quantitative evaluation of the carbon-based chemical species may be ambiguous, since many distinctive functional groups may possess very similar binding energies. Furthermore, differentiation between energies arising from either a defect or oxygen-based chemical specie may be very troublesome. Therefore, many researchers suggest supporting the $\mathrm{C} 1 s$ evaluation with a deconvolution of the oxygen band. This is backed up by a very high probability that all of the oxygen atoms,
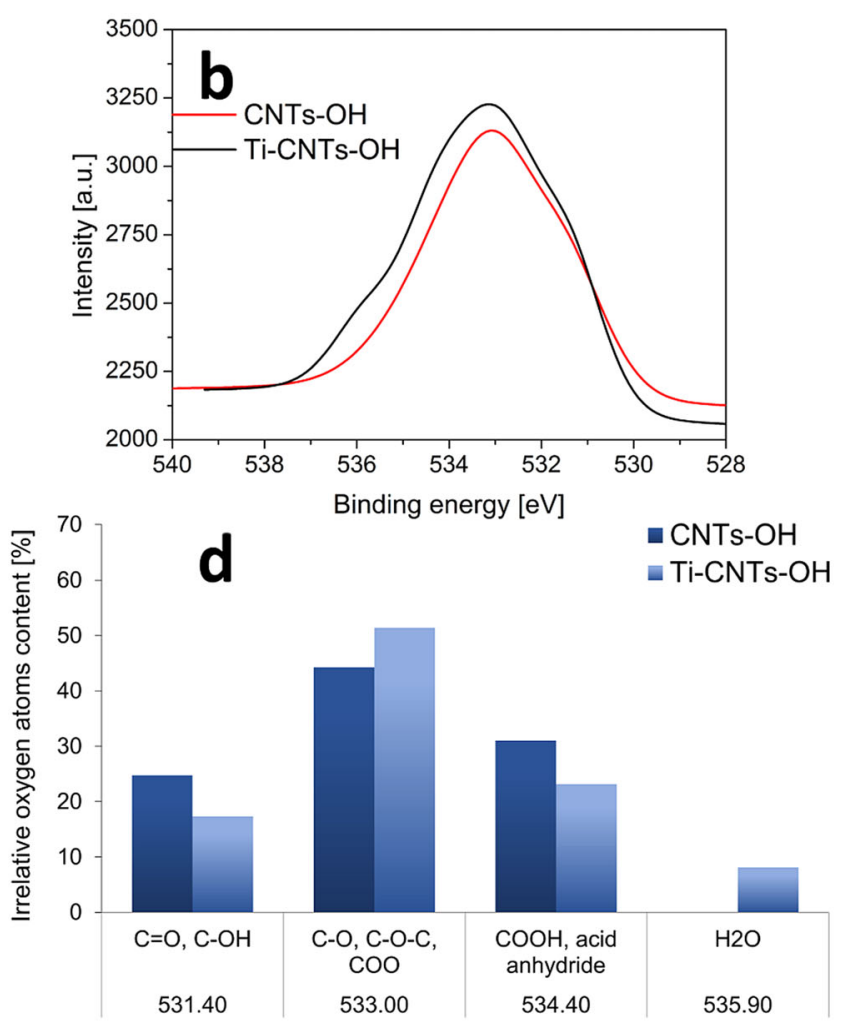

evaluation of the deconvoluted $\mathrm{C} 1 s$ (c) and $\mathrm{O} 1 s$ (d) bands, found in the spectra of the tested samples

present in the sample, arise from functional groups present on the sidewalls of the CNTs. Thus, this approach is expected to provide a more accurate knowledge on the amount and type of the oxygen-based chemical species. A quantitative evaluation of the oxygen-based species is given in Fig. 7d. It can be seen that in the Ti-CNTs-OH, an amount of the $\mathrm{C}=\mathrm{O}$ and $\mathrm{C}-\mathrm{OH}$ groups was smaller $(17.3 \%)$ than in the CNTs-OH $(24.8 \%)$ [20, 29]. This suggests that the increased intensity of the $285.8 \mathrm{eV}$ in the Ti-CNTs-OH is attributed mostly to the presence of structural defects, instead of functional groups. At the same time, an increased intensity of the peak at $533 \mathrm{eV}$ was observed, indicating presence of higher amount of various COO species and ether [20], suggesting interaction of some of the functional groups at the cost of carboxyl groups [35].

It is hard to unequivocally point out the main reason for different chemical composition of the CNTs deposits, when compared to the starting material. We believe that there might be at least three causes for that. First of all, in the starting material, we have a population of assorted CNTs, some of which do not have a sufficient mobility under the influence of the electric current and are not deposited on the surface of titanium. Thus, the EPD fractionates the tubes and only CNTs of selected amount and type of 
functional groups are deposited. Secondly, one must consider CNTs flocculation that may induce interactions between the functional groups and change the overall electric charge of the CNTs. As a consequence, chemical composition of the tubes in a dilution can be very different than in the starting material. Finally, the CNTs can be forced to interact with one another under the influence of the electric current, causing formation of new functional groups, and stress induces creation of defects.

\subsection{In vitro cell culture experiments}

In the present work, WST-8 test was performed to evaluate the cytotoxic effect of the tested sample extracts on human foetal osteoblasts (hFOB 1.19) after 24- and 48-h exposure. The extracts were found not to affect cell viability throughout the full length of the experiment as near $100 \%$ metabolic activity of the hFOB cells compared to the PS extract-treated control cells was observed (Fig. 8). According to the ISO 10993-5, a sample that has no cytotoxic effect on cells might be regarded as biocompatible [12].

Live/dead double fluorescence staining confirmed results obtained with WST- 8 test and proved that the $\mathrm{CNTs}-\mathrm{OH}$ coating did not affect cell viability. Fluorescence laser scanning microscope observation showed good osteoblast growth on both titanium and carbon nanotube surfaces (Fig. 9). Only small amount of dead cells (red fluorescence) was observed on both samples. Moreover, $48 \mathrm{~h}$ after cell seeding osteoblasts were well spread and had lengthened shape, indicating their good adhesion to the tested surfaces.

Cell morphology analysis using fluorescence laser scanning microscope revealed that osteoblasts seeded on

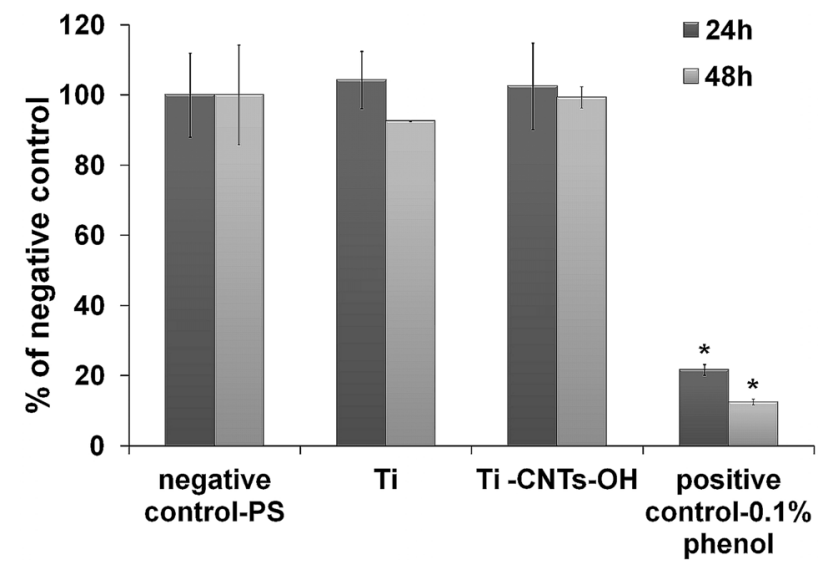

Fig. 8 Cytotoxicity evaluation of the tested sample extracts, using hFOB 1.19 cells by means of WST-8 assay; the values for the PS extract-treated control cells and the sample extract-treated cells were significantly different according to the unpaired $t$ test $(* P<0.05)$ both, Ti and Ti-CNTs-OH, specimens were well spread, flattened, had typical lengthened shape and extensive cytoskeletal structure (Fig. 10). Moreover, after 48-h incubation, there were similar amount of cells on both tested samples. However, osteoblasts cultured on the TiCNTs-OH surface had noticeably smaller and deformed nuclei (Fig. 10b). Some recent papers demonstrated that micro-structured surfaces may cause nucleus deformation $[37,38]$ which does not affect cell viability, proliferation and differentiation [38]. Thus, it may be inferred that observed abnormal morphology of nuclei was caused by specific Ti-CNTs-OH micro-topography resulting from uniformly distributed CNT layer covered with randomly but evenly distributed larger agglomerates.

According to available literature, osteoblast adhesion and proliferation is highly dependent on the surface properties of the biomaterial like surface chemistry and charge [39] as well as wettability and topography [40]. Osteoblasts reveal better growth on polar nanorough surfaces with lower water contact angle (higher wettability) compared to the nanosmooth materials with higher water contact angle (lower wettability) [40]. Thus, it may be concluded that good osteoblast adhesion and growth on the Ti-CNTs-OH resulted from its nanorough surface (Fig. 5d) and relatively high wettability, slightly higher compared to the Ti sample (Fig. 11) as proven in our recent report [7]. Moreover, in our previous study it was demonstrated that $\mathrm{CNTs}-\mathrm{OH}$ layer increases the total surface energy of $\mathrm{Ti}$, whereas it slightly decreases the polar part compared to the Ti sample-probably due to the small amount of polar groups and increased nanoroughness [7].

In our other research [6], it was proved that CNT layer deposited for $30 \mathrm{~s}$ from water ( $\mathrm{Ti}-\mathrm{CNTs}-\mathrm{H}_{2} \mathrm{O}$ ) possessed comparable non-cytotoxicity to the $\mathrm{Ti}-\mathrm{CNTs}-\mathrm{OH}$, but was found to be less favourable to osteoblast adhesion, spreading and growth. This observation is most likely a result of significantly different chemical composition of previously tested sample ( $\left.\mathrm{Ti}-\mathrm{CNTs}-\mathrm{H}_{2} \mathrm{O}\right)$. In mentioned study, a densely packed and highly oxidized layer of CNTs, with a large share of $\mathrm{C}=\mathrm{O}$ groups and over two times higher amount of oxygen-based functional groups, was reported to decrease the level of osteoblast spreading and proliferation, while still being biocompatible [6] Herein, the surface was formed of more randomly and loosely distributed tubes, with a significantly lower amount of oxygen, mainly observed by the reduction of the $\mathrm{C}=\mathrm{O}$ species, and these properties were found to be favourable to osteoblast survival, adhesion and spreading. Thus, proposed within this study EPD of CNTs on titanium surfaces may allow obtaining biocompatible materials for biomedical applications as orthopaedic permanent implants whenever covering by bone tissue cells is desired. 
Fig. 9 Fluorescence laser scanning microscope images of cells growing on the surface of $\mathrm{Ti}(\mathbf{a}, \mathbf{c})$ and Ti-CNTs-OH (b, d) after live/dead staining; images were obtained after $24-\mathrm{h}$ (a, b) and 48-h (c,

d) incubation; cytoplasm of viable cells was stained with calcein-AM dye (green fluorescence), and nucleic acids of dead cells were stained with PI dye (red fluorescence); magn. $100 \times$

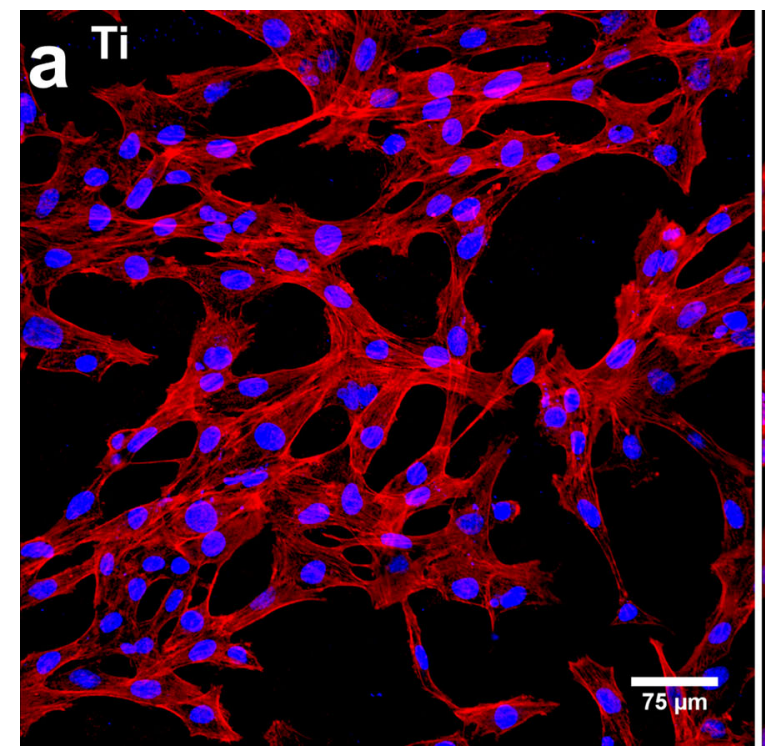

Fig. 10 Fluorescence laser scanning microscope observation of cells cultured on the surface of Ti (a) and Ti-CNTs-OH (b) samples $48 \mathrm{~h}$ after cell seeding; F-actin filaments of cytoskeleton were stained with
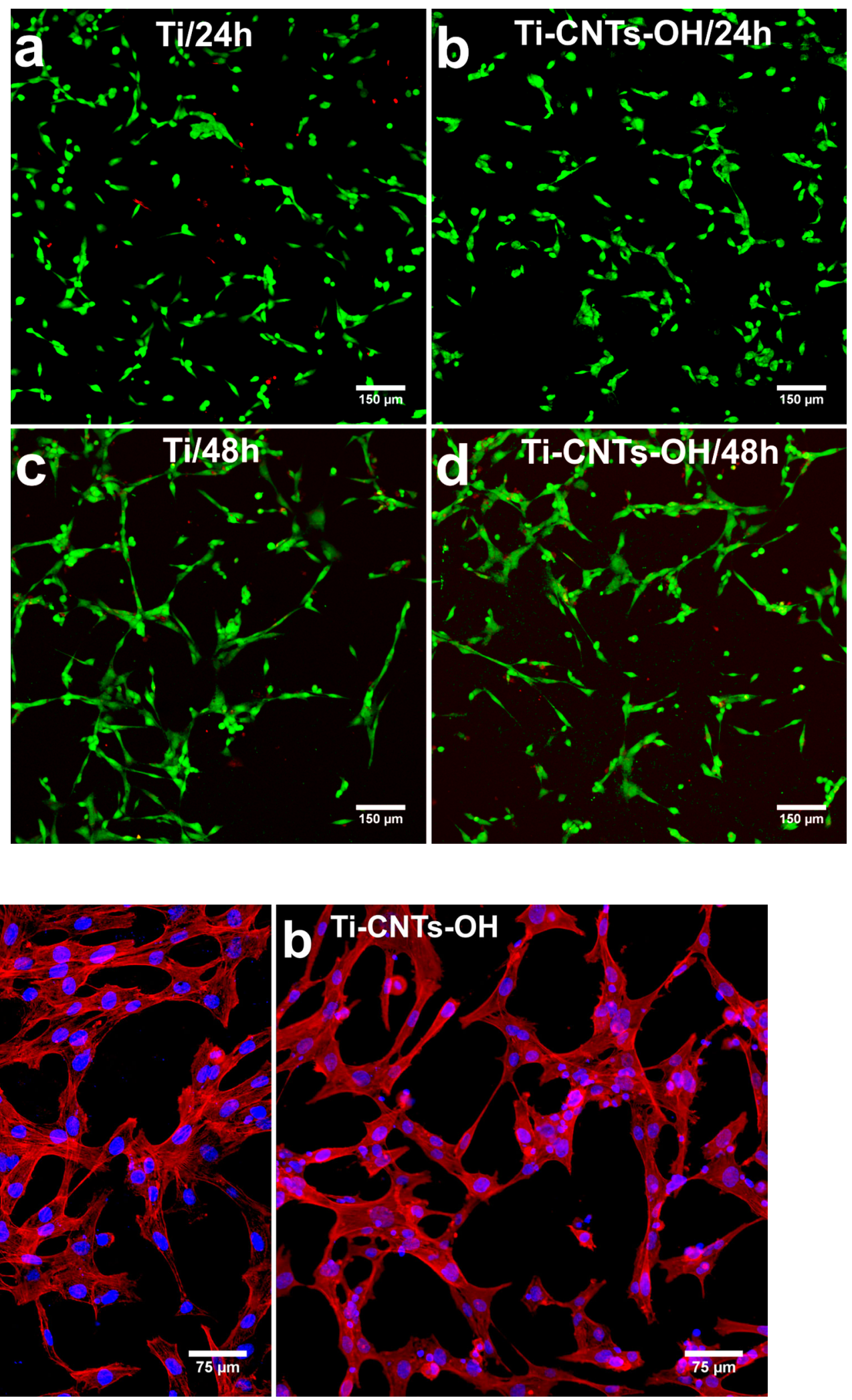

AlexaFluor635phalloidin (red fluorescence), and the nuclei were stained with Hoechst 33342 (blue fluorescence); magn. 200× 
a

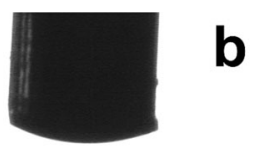

$\mathrm{Ti}$

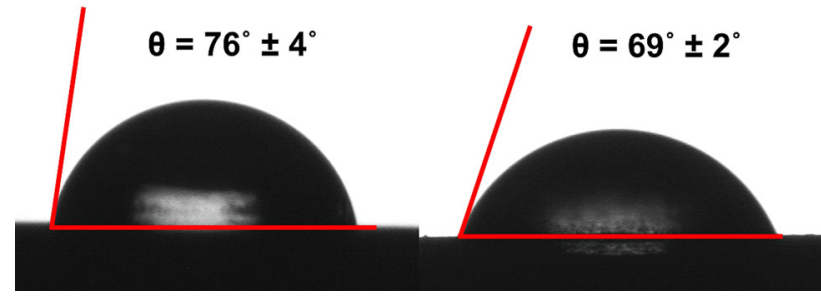

Fig. 11 Images presenting water contact angle measurement for $\mathrm{Ti}$ (a) and Ti-CNTs-OH (b) samples

The aim of the study was to fabricate and extensively characterize a layer of carbon nanotubes deposited on the surface of titanium by electrophoretic deposition process. In the research, we reported two major novelties in the field of the electrophoretic deposition of the CNTs-first of all, we used commercially available CNTs with no additional chemical treatment. This might provide higher repeatability of the results and might be the first step in up-scaling the process. Secondly, a novel composition of solvents was applied to form a stable suspension of CNTs. This suspension was found to have a large charging capability and particles mobility, resulting in stable kinetics of deposition and a high yield of deposits. These promising results might be the first step in up-scaling the process of the EPD of the CNTs.

\section{Conclusion}

EPD can be used to fabricate CNT layers of different properties, depending on the parameters of the process, designed to meet specific criteria for the targeted application, including biomedical. Influence on cells will depend on the chemistry and nanotopography of the surface and those parameters can be controlled and the process can be designed in such a way that desired specifications are met. Conducted research presented herein resulted in successful fabrication of CNT layer that is found to be supportive for osteoblast growth. By comparison with our other studies [6], it was proved that by selecting proper type of CNTs and altering different parameters of the process, electrophoretic deposition of CNTs on materials may be reproducible simple method to obtain products having different influence on osteoblasts-either inhibiting [6] or favouring their adhesion and survival. Thus, by carefully tailoring the process, one can obtain CNTs-based materials that could find application in different fields of biomedicine-as permanent implants that should be covered by host tissue or temporary implantable devices in which covering by connective tissue is not a desired phenomenon.

Acknowledgments This work has been supported by the National Science Centre (NCN) Grants Nos.: UMO-2014/13/B/ST8/01195 and UMO-2013/11/N/ST8/01357, and developed using the equipment purchased within the agreement No. PORPW.01.03.00-06-010/09-00 Operational Programme Development of Eastern Poland 2007-2013, Priority Axis I, Modern Economy, Operations 1.3. Innovations Promotion.

Open Access This article is distributed under the terms of the Creative Commons Attribution 4.0 International License (http://crea tivecommons.org/licenses/by/4.0/), which permits unrestricted use, distribution, and reproduction in any medium, provided you give appropriate credit to the original author(s) and the source, provide a link to the Creative Commons license, and indicate if changes were made.

\section{References}

1. B.G. Demczyk, Y.M. Wang, J. Cumings, M. Hetman, W. Han, A. Zettl, R.O. Ritchie, Mater. Sci. Eng. A Struct. 334, 173-178 (2002)

2. Z.D. Han, A. Fina, Prog. Polym. Sci. 36, 914-944 (2011)

3. B. Ferrari, R. Moreno, J. Eur. Ceram. Soc. 30, 1069-1078 (2010)

4. L. Besra, M. Liu, Prog. Mater. Sci. 52, 1-61 (2007)

5. C.S. Du, D. Heldbrant, N. Pan, Mater. Lett. 57, 434-438 (2002)

6. A. Przekora, A. Benko, M. Nocun, J. Wyrwa, M. Blazewicz, G. Ginalska, Mater. Sci. Eng. C Mater. Biol. Appl. 45, 287-296 (2014)

7. A. Benko, A. Frączek-Szczypta, E. Menaszek, J. Wyrwa, M. Nocuń, M. Błażewicz, J. Mater. Sci.: Mater. Med. 26, 1-13 (2015)

8. J. Beuvelot, C. Bergeret, R. Mallet, V. Fernandez, J. Cousseau, M.F. Basle, D. Chappard, Acta Biomater. 6, 4110-4117 (2010)

9. Nanostructured \& Amorphous Materials, Inc, Amorphous Products I Nanoscale Products - Short MWNT-OH (95+\%, OD 10-20 nm) (2015), http://www.nanoamor.com/inc/sdetail/27326. Accessed 25 Sept 2015

10. S.F. Lamolle, M. Monjo, M. Rubert, H.J. Haugen, S.P. Lyngstadaas, J.E. Ellingsen, Biomaterials 30, 736-742 (2009)

11. Y. Li, S. Zou, D. Wang, G. Feng, C. Bao, J. Hu, Biomaterials 31, 3266-3273 (2010)

12. ISO 10993-5:2009, Biological evaluation of medical devices Part 5: Tests for in vitro cytotoxicity. International Organization for Standardization (2009), http://www.iso.org/iso/catalogue_ detail.htm?csnumber $=36406$

13. P. Sarkar, D. De, T. Uchikochi, L. Besra, Electrophoretic deposition (EPD): fundamentals and novel applications in fabrication of advanced ceramic microstructures, in Electrophoretic Deposition of Nanomaterials, ed. by J.H. Dickerson, A.R. Boccaccini (Springer, New York, 2012), pp. 181-215

14. R.V. Goreham, A. Mierczynska, L.E. Smith, R. Sedev, K. Vasilev, RSC Adv. 3, 10309-10317 (2013)

15. R.A. DiLeo, B.J. Landi, R.P. Raffaelle, J. Appl. Phys. 101, 064307 (2007). doi:10.1063/1.2712152

16. M.S. Dresselhaus, G. Dresselhaus, R. Saito, A. Jorio, Phys. Rep. 409, 47-99 (2005)

17. C. Thomsen, S. Reich, J. Maultzsch, Philos. Trans. Ser. A Math. Phys. Eng. Sci. 362, 2337-2359 (2004)

18. M.S. Dresselhaus, A. Jorio, A.G. Souza Filho, R. Saito, Philos. Trans. Ser. A Math. Phys. Eng. Sci. 368, 5355-5377 (2010) 
19. O. Beyssac, B. Goffe, J.P. Petitet, E. Froigneux, M. Moreau, J.N. Rouzaud, Spectrochim. Acta Part A Mol. Biomol. Spectrosc. 59, 2267-2276 (2003)

20. V. Datsyuk, M. Kalyva, K. Papagelis, J. Parthenios, D. Tasis, A. Siokou, I. Kallitsis, C. Galiotis, Carbon 46, 833-840 (2008)

21. A. Wesełucha-Birczyńska, M. Łabanowska, M. Kurdziel, M. Filek, Vib. Spectrosc. 60, 113-117 (2012)

22. A. Jorio, M.A. Pimenta, A.G. Souza, R. Saito, G. Dresselhaus, M.S. Dresselhaus, New J. Phys. 5, 139.1-139.17 (2003). doi:10. 1088/1367-2630/5/1/139

23. N.F. Andrade, D.S.T. Martinez, A.J. Paula, J.V. Silveira, O.L. Alves, A.G. Souza, J. Nanopart. Res. 15, 1761 (2013)

24. A. Fraczek-Szczypta, E. Dlugon, A. Weselucha-Birczynska, M. Nocun, M. Blazewicz, J. Mol. Struct. 1040, 238-245 (2013)

25. L.G. Cancado, A. Jorio, E.H. Ferreira, F. Stavale, C.A. Achete, R.B. Capaz, M.V. Moutinho, A. Lombardo, T.S. Kulmala, A.C. Ferrari, Nano Lett. 11, 3190-3196 (2011)

26. D. Liu, P.E.J. Flewitt, in Spectroscopic Properties of Inorganic and Organometallic Compounds, chap. 5, vol. 45, ed. by R. Douthwaite, S. Duckett, J. Yarwood,(The Royal Society of Chemistry, 2014), pp. 141-177. doi:10.1039/978178262148500141

27. B. Wincheski, J.-W. Kim, G. Sauti, E. Wainwright, P. Williams, E.J. Siochi, Quantiative Nondestructive Evaluation (QNDE) Review Conference, (Iowa State Univ., Center for Nondestructive Evaluation, Ames, Boise, 2014)

28. Z. Liu, J. Zhang, B. Gao, Chem. Commun. (45), 6902-6918 (2009)
29. M.T. Martinez, M.A. Callejas, A.M. Benito, M. Cochet, T. Seeger, A. Anson, J. Schreiber, C. Gordon, C. Marhic, O. Chauvet, J.L.G. Fierro, W.K. Maser, Carbon 41, 2247-2256 (2003)

30. W. Xia, Y. Wang, R. Bergsträßer, S. Kundu, M. Muhler, Appl. Surf. Sci. 254, 247-250 (2007)

31. G. Moraitis, Z. Špitalský, F. Ravani, A. Siokou, C. Galiotis, Carbon 49, 2702-2708 (2011)

32. K. Wepasnick, B. Smith, J. Bitter, D. Howard Fairbrother, Anal. Bioanal. Chem. 396, 1003-1014 (2010)

33. A. Pacuła, R.P. Socha, M. Zimowska, M. Ruggiero-Mikołajczyk, D. Mucha, P. Nowak, Appl. Clay Sci. 72, 163-174 (2013)

34. Y. Xing, L. Li, C.C. Chusuei, R.V. Hull, Langmuir: ACS J. Surf. Colloids 21, 4185-4190 (2005)

35. X. Ling, Y. Wei, L. Zou, S. Xu, Appl. Surf. Sci. 276, 159-166 (2013)

36. Y.C. Chiang, W.H. Lin, Y.C. Chang, Appl. Surf. Sci. 257, 2401-2410 (2011)

37. F. Badique, D.R. Stamov, P.M. Davidson, M. Veuillet, G. Reiter, J.-N. Freund, C.M. Franz, K. Anselme, Biomaterials 34, 2991-3001 (2013)

38. P. Davidson, H. Özçelik, V. Hasirci, G. Reiter, K. Anselme, Adv. Mater. 21(35), 3586 (2009). doi:10.1002/adma.200900582

39. X. Le, G.E. Poinern, N. Ali, C.M. Berry, D. Fawcett, Int. J. Biomater. (2013). doi:10.1155/2013/782549

40. H.-I. Chang, Y. Wang, in Regenerative Medicine and Tissue Engineering: Cells and Biomaterials, ed. by D. Eberli (InTech Open Access Publisher, Rijeka, 2011). doi:10.5772/21983 